C-A/AP/\#165 September 2004

\title{
Linear coupling parameterization in the betatron phase rotation frame
}

\author{
Yun Luo
}

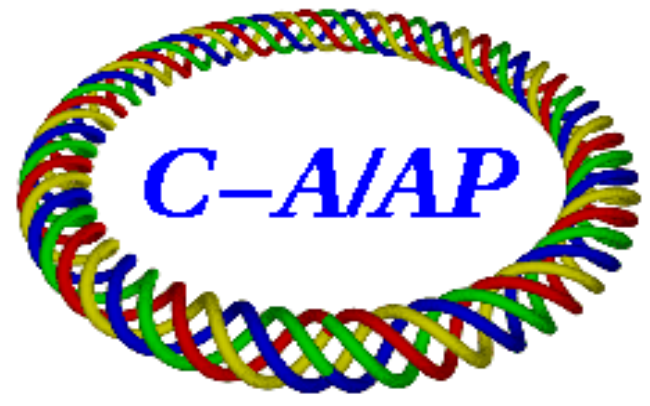

Collider-Accelerator Department Brookhaven National Laboratory Upton, NY 11973 
$\mathrm{CAD} / \mathrm{AP} / 165$

September 1, 2004

\title{
Linear coupling parameterization in the betatron phase rotation frame
}

\author{
Yun Luo \\ Brookhaven National Laboratory, Upton, NY 11973, USA
}

Following Ripken's linear coupling parameterization procedure, the linear coupling parameterization in the betatron phase rotation frame is introduced. In the parameterization, the two eigenmodes' betatron oscillation phases and phase advances are well defined. The general expression of the coordinates $\left(x, x^{\prime}, y, y^{\prime}\right)$ in the laboratory frame are given independent of different beam excitations.

The transfer matrix $\mathbf{P}$ from the betatron phase rotation frame to the laboratory frame are discussed in detail. It can be obtained from the eigenvectors of the one-turn transfer map $\mathbf{T}$. It also has tight connections to Twiss and coupling parameters defined in Edwards-Teng's parameterization. It can be constructed from BPM turn-by-turn data with the precisely measured eigentunes and betatron initial phases.

The phase ellipses and beam sizes in different projection planes are easily obtained in the betatron phase rotation parameterization. The physical meanings of the coupling parameter $r$ and the coupling matrix $\mathbf{C}$ defined in Edwards-Teng's parameterization become clear.

The two eigenmode betatron phase advances, the matrix $\mathbf{P}$, therefore Twiss and coupling parameters at point $s_{2}$ are described in terms of the section transfer map $\mathbf{T}_{\mathbf{1 \rightarrow} \mathbf{2}}$ and Twiss and coupling parameters at the starting point $s_{1}$.

The linear coupling betatron phase rotation parameterization is useful for the turn-by-turn BPM data interpretation. The three dimensional linear coupling parameterization in the betatron phase rotation frame can be obtained in the same way as the two-dimensional parameterization. And the parameterization using $(x, p x, y, p y)$ canonical coordinates is similar to that shown here with $\left(x, x^{\prime}, y, y^{\prime}\right)$ coordinates. The betatron phase rotation parameterization can be adjusted for the transport line linear coupling research purpose, too.

\section{Introduction}

The Courant-Snyder parameterization [1] of the uncoupled one-dimensional particle's betatron oscillation in the circular accelerators has been proved to be a great success. The pursuit to cast the coupled twodimensional transverse betatron motion into an elegant way as the one-dimensional parameterization is continuing. Here we give another approach in its sort.

There are two main directions to parameterize the two-dimensional linearly coupled betatron motions. One is to start from the eigenvectors of the one-turn $4 \times 4$ transfer matrix to construct the particle's four coordinates in the laboratory frame. The eigenvectors transfer from one point to another in the ring so that the coordinates in the eigenvector frame are constant. Comparing to the one-dimensional Courant-Snyder parameterization, new Twiss parameters in the two-dimensional situation are defined accordingly. One wellknown parameterization in this sort was achieved by G. Ripken $[2,3,4]$. There, four $\beta$ and four $\alpha$ functions are defined. Knowing the expression of the four coordinates in the laboratory frame, the phase ellipses and beam sizes into different observation planes are easily obtained.

Another approach is to directly decouple the one-turn transfer map in the laboratory frame into an uncoupled $4 \times 4$ one-turn map through the matrix similarity transformation. One successful approach in this direction had been obtained by Edwards and Teng $[5,6]$ and improved by others $[7,8,9,10]$. The parameterization is proved successful. There two sets of Twiss parameters from two eigenmodes are defined and a coupling matrix is introduced. It has been widely used in coupling measurements, where the normalized $\overline{\mathbf{C}}$ is used $[11,9]$.

Comparing the two successful parameterizations by Ripken, Edwards and Teng, we find that Ripken's parameterization give straight-forward expression of the particle's coordinates in the laboratory frame. There the particle motion's phases and phase advances between two points are both well defined. The shortcoming 
of this approach is that it is a little complicated. The inverse transfer from its defined Twiss parameters to the one-turn transfer map hasn't been found [12].

Edwards-Teng's parameterization is based on the matrix manipulations. The transfers between its defined Twiss, coupling parameters and the one-turn map are easily obtained. The unsolved problem of the parameterization is its ambiguous definitions of betatron phases and phase advances. The general expression of one particle's motion in the laboratory frame is not given. So it is not used to calculate the phase ellipses, phase areas and beam sizes in different projection planes. The relation between the one-turn transfer map's eigenvectors and its defined Twiss and coupling parameters are not given.

With high performance digital BPMs and the modern analytical techniques $[11,13,14,15,16,17,18]$, the betatron phase advances can be very precisely measured in the beam experiments. The merit of the phase measurement is that it is independent from the BPM position offsets and their gains. It has attracted much attention in how to use them in the past few years [19]. For a specific betatron oscillation, the two eigenmodes' phases and phase advances are all well defined. To include them into the linear coupling parameterizations are demanded. This article will give an approach to tackle those mysteries.

Following the procedure of Ripken's parameterization, we transfer the four coordinates in the laboratory frame to the betatron phase rotation frame. Each laboratory coordinate will have four terms. Each eigenmode contributes two terms. It will be found that the transfer matrix from the laboratory frame to the betatron phase rotation frame can be obtained simply from the eigenvectors of the one-turn map in the laboratory frame. The projection phase ellipses and their areas, the coupling tilt angle in the $x-y$ plane are also easily obtained. The betatron phase rotation parameterization is self-consistent formalism. It is found that the transfer matrix from the laboratory frame to the betatron phase rotation frame have very tight connections to Twiss and coupling parameters defined in Edwards-Teng's parameterization. So they are directly borrowed into the betatron phase rotation parameterization.

The article is organized in the following way: first we introduce the phase rotation frame starting from Ripken's parameterization but with different expression of the four laboratory coordinates. Then we discuss how to construct the matrix $\mathbf{P}$ analytically and experimentally. The phase ellipses and their areas, and the beam sizes are given. The propagation of the matrix $\mathbf{P}$ in terms of the section transfer matrix $\mathbf{T}_{\mathbf{1} \rightarrow \mathbf{2}}$ are obtained.

\section{Phase rotation frame}

In the general linearly coupled situation, it is known that the displacements $x$ and $y$ include the contributions from both eigenmodes. we assume that mode I is more linked to the horizontal plane, and mode II is more linked to the vertical plane. The $n$th turn's $x$ and $y$ can be casted as:

$$
\left\{\begin{array}{l}
x_{n}=A_{1,1} \cos \left(2 \pi \mu_{1}(n-1)+\phi_{1,1}\right)+A_{1,2} \cos \left(2 \pi \mu_{2}(n-1)+\phi_{1,2}\right) \\
y_{n}=A_{2,1} \cos \left(2 \pi \mu_{1}(n-1)+\phi_{2,1}\right)+A_{2,2} \cos \left(2 \pi \mu_{2}(n-1)+\phi_{2,2}\right)
\end{array},\right.
$$

where the amplitude coefficients $A$ and initial phases $\phi$ both have subscripts $(i, j)$. The first subscript represents the horizontal or vertical plane. The second subscript represents mode I or mode II.

\section{$2.14 \times 4 \mathbf{P}$ matrix}

Eq. (1) has a very clear physical meaning. The tunes $\mu_{1,2}$ and the initial phase $\phi_{i, j}$ can be precisely obtained from BPM turn-by-turn data in beam experiments. They are all well defined quantities for a specific betatron motion. The angles $x^{\prime}$ and $y^{\prime}$ also can be casted into two modes' contributions like Eq. (1). However, the shortcoming of this parameterization is that there are two many $A \mathrm{~s}$ and $\phi \mathrm{s}$ and no clear relations between them.

One simple alternative is to cast the coordinates with the action-angle variables as the uncoupled onedimensional situation [1], where $x$ and $y$ are:

$$
\left\{\begin{array}{l}
x=\sqrt{2 J_{x} \beta_{x}} \cos \Phi_{x} \\
y=\sqrt{2 J_{y} \beta_{y}} \cos \Phi_{y}
\end{array}\right.
$$

where $J_{x, y}$ are the horizontal and vertical actions, $\Phi_{x}$ and $\Phi_{y}$ are the phases of the betatron oscillations. By taking differentials of the above two equations, we obtain two terms for each $x^{\prime}$ or $y^{\prime}$ if we still keep the betatron phases $\Phi_{x, y}$. 
So in the linearly coupled case, if we keep the two eigenmodes' phases, there will probably be four terms for each coordinate of $\left(x, x^{\prime}, y, y^{\prime}\right)$ at one point in the ring, then we get:

$$
\begin{gathered}
\left(\begin{array}{c}
x \\
x^{\prime} \\
y \\
y^{\prime}
\end{array}\right)=\mathbf{P} \cdot\left(\begin{array}{c}
\cos \Phi_{1} \\
\sin \Phi_{1} \\
\cos \Phi_{2} \\
\sin \Phi_{2}
\end{array}\right), \\
\mathbf{P}=\left(\begin{array}{llll}
p_{11} & p_{12} & p_{13} & p_{14} \\
p_{21} & p_{22} & p_{23} & p_{24} \\
p_{31} & p_{32} & p_{33} & p_{34} \\
p_{41} & p_{42} & p_{43} & p_{44}
\end{array}\right),
\end{gathered}
$$

where $\Phi_{1,2}$ are the two eigenmodes' betatron oscillation phases. The one turn's betatron phase advances are $2 \pi \mu_{1}$ for mode I, $2 \pi \mu_{2}$ for mode II. Eq. (3) looks like that from Ripken's expression of $\left(x, x^{\prime}, y, y^{\prime}\right)$ coordinates. However, here we include four terms for each coordinate, and only two betatron phases are introduced.

\subsection{Phase rotations}

Here we give the frequently used phase rotation matrix in accelerator physics:

$$
\begin{gathered}
\mathbf{R}\left(\boldsymbol{\Delta} \boldsymbol{\Phi}_{\mathbf{i}}\right)=\left(\begin{array}{cc}
\cos \left(\Delta \Phi_{i}\right) & \sin \left(\Delta \Phi_{i}\right) \\
-\sin \left(\Delta \Phi_{i}\right) & \cos \left(\Delta \Phi_{i}\right)
\end{array}\right) \\
\mathbf{R}\left(\boldsymbol{\Delta} \boldsymbol{\Phi}_{\mathbf{1}}, \boldsymbol{\Delta} \boldsymbol{\Phi}_{\mathbf{2}}\right)=\left(\begin{array}{cc}
\mathbf{R}\left(\boldsymbol{\Delta} \boldsymbol{\Phi}_{\mathbf{1}}\right) & 0 \\
0 & \mathbf{R}\left(\boldsymbol{\Delta} \boldsymbol{\Phi}_{\mathbf{2}}\right)
\end{array}\right) .
\end{gathered}
$$

Careful readers will notice that both of them are clockwise rotation matrix. In order to get the phase advance propagation like $\left.\Phi_{1,2}\right|_{s 2}=\left.\Phi_{1,2}\right|_{s 1}+\Delta \Phi_{1,2}$, we change the signs of the sin functions in Eq. (3):

$$
\left(\begin{array}{c}
x \\
x^{\prime} \\
y \\
y^{\prime}
\end{array}\right)=\left(\begin{array}{llll}
p_{11} & p_{12} & p_{13} & p_{14} \\
p_{21} & p_{22} & p_{23} & p_{24} \\
p_{31} & p_{32} & p_{33} & p_{34} \\
p_{41} & p_{42} & p_{43} & p_{44}
\end{array}\right) \cdot\left(\begin{array}{c}
\cos \Phi_{1} \\
-\sin \Phi_{1} \\
\cos \Phi_{2} \\
-\sin \Phi_{2}
\end{array}\right)
$$

It is easily to prove:

$$
\left(\begin{array}{l}
\cos \left(\Phi_{1}+\Delta \Phi_{1}\right) \\
-\sin \left(\Phi_{1}+\Delta \Phi_{1}\right) \\
\cos \left(\Phi_{2}+\Delta \Phi_{2}\right) \\
-\sin \left(\Phi_{2}+\Delta \Phi_{2}\right)
\end{array}\right)_{2}=\mathbf{R}\left(\boldsymbol{\Delta} \Phi_{\mathbf{1}}, \boldsymbol{\Delta} \boldsymbol{\Phi}_{\mathbf{2}}\right) \cdot\left(\begin{array}{c}
\cos \left(\Phi_{1}\right) \\
-\sin \left(\Phi_{1}\right) \\
\cos \left(\Phi_{2}\right) \\
-\sin \left(\Phi_{2}\right)
\end{array}\right)_{\mathbf{1}}
$$

So the coordinates in the betatron phase rotation frame are rotating with the two eigenmodes' phase advances when the particle travels along the ring. The amplitude of coordinates in the rotation frame are constant.

\subsection{Actions $J_{1}$ and $J_{2}$}

By now the elements of $\mathbf{P}$ are related with the particle's oscillation amplitudes or energy. In order to make $p_{i j}$ independent of individual particles, here we introduce actions into. In the uncoupled situation, the action $J_{1}$ of mode I will appear in the left upper block of $\mathbf{P}$. Action $J_{2}$ of mode II will appear in the right lower block of $\mathbf{P}$. Then we modify Eq. (7) as:

$$
\left(\begin{array}{c}
x \\
x^{\prime} \\
y \\
y^{\prime}
\end{array}\right)=\left(\begin{array}{llll}
p_{11} & p_{12} & p_{13} & p_{14} \\
p_{21} & p_{22} & p_{23} & p_{24} \\
p_{31} & p_{32} & p_{33} & p_{34} \\
p_{41} & p_{42} & p_{43} & p_{44}
\end{array}\right) \cdot\left(\begin{array}{c}
\sqrt{2 J_{1}} \cos \Phi_{1} \\
-\sqrt{2 J_{1}} \sin \Phi_{1} \\
\sqrt{2 J_{2}} \cos \Phi_{2} \\
-\sqrt{2 J_{2}} \sin \Phi_{2}
\end{array}\right) .
$$

The propagation of the coordinates in the betatron phase rotation frame is :

$$
\left(\begin{array}{l}
\sqrt{J_{1}} \cos \left(\Phi_{1}+\Delta \Phi_{1}\right) \\
-\sqrt{J_{1}} \sin \left(\Phi_{1}+\Delta \Phi_{1}\right) \\
\sqrt{J_{2}} \cos \left(\Phi_{2}+\Delta \Phi_{2}\right) \\
-\sqrt{J_{2}} \sin \left(\Phi_{2}+\Delta \Phi_{2}\right)
\end{array}\right)_{2}=\mathbf{R}\left(\boldsymbol{\Delta} \Phi_{1}, \Delta \Phi_{2}\right) \cdot\left(\begin{array}{c}
\sqrt{J_{1}} \cos \left(\Phi_{1}\right) \\
-\sqrt{J_{1}} \sin \left(\Phi_{1}\right) \\
\sqrt{J_{2}} \cos \left(\Phi_{2}\right) \\
-\sqrt{J_{2}} \sin \left(\Phi_{2}\right)
\end{array}\right)_{\mathbf{1}} .
$$




\subsection{Betatron phases $\Phi_{1}$ and $\Phi_{2}$}

In Eq. (10) there is still an ambiguity in the definitions of eigenmodes' phases $\Phi_{1,2}$. In principle, one can define arbitrary $\Phi_{1,2}$ in Eq. (10), there still will be a constant $\mathbf{P}$ at one point in the ring.

We notice that in the uncoupled one-dimensional situation, Courant and Snyder defined the displacement's phase as the eigenmode's phase. Therefore there is only one term for the displacement coordinate. Then $x^{\prime}$ or $y^{\prime}$ has two terms if we keep the displacement's phase. Among them, one term is in-phase to the displacement, another is out-of-phase to the displacement.

Here we forcedly define $\Phi_{1}$ as the phase of mode I 's contribution to $x$ displacement, and $\Phi_{2}$ as the phase of mode II's contribution to $y$ displacement. Therefore, the element $p_{12}$ and $p_{34}$ in matrix $\mathbf{P}$ equal to zero:

$$
\left(\begin{array}{c}
x \\
x^{\prime} \\
y \\
y^{\prime}
\end{array}\right)=\left(\begin{array}{cccc}
p_{11} & 0 & p_{13} & p_{14} \\
p_{21} & p_{22} & p_{23} & p_{24} \\
p_{31} & p_{32} & p_{33} & 0 \\
p_{41} & p_{42} & p_{43} & p_{44}
\end{array}\right) \cdot\left(\begin{array}{c}
\sqrt{2 J_{1}} \cos \Phi_{1} \\
-\sqrt{2 J_{1}} \sin \Phi_{1} \\
\sqrt{2 J_{2}} \cos \Phi_{2} \\
-\sqrt{2 J_{2}} \sin \Phi_{2}
\end{array}\right) .
$$

The eigenmode phases at one point in the ring are:

$$
\left\{\begin{array}{l}
\Phi_{1}=2 \pi \mu_{1}(n-1)+\phi_{1,0} \\
\Phi_{2}=2 \pi \mu_{2}(n-1)+\phi_{2,0}
\end{array},\right.
$$

$\phi_{1,0}$ and $\phi_{2,0}$ are the initial betatron phases for the two eigenmodes at that point. They can be precisely measured experimentally from the turn-by-turn BPM data.

Each coordinate in the laboratory frame is then given :

$$
\left\{\begin{array}{c}
x=p_{11} \sqrt{2 J_{1}} \cos \Phi_{1}+p_{13} \sqrt{2 J_{2}} \cos \Phi_{2}-p_{14} \sqrt{2 J_{2}} \sin \Phi_{2} \\
x^{\prime}=p_{21} \sqrt{2 J_{1}} \cos \Phi_{1}-p_{22} \sqrt{2 J_{1}} \sin \Phi_{1}+p_{23} \sqrt{2 J_{2}} \cos \Phi_{2}-p_{24} \sqrt{2 J_{2}} \sin \Phi_{2} \\
y=p_{31} \sqrt{2 J_{1}} \cos \Phi_{1}-p_{32} \sqrt{2 J_{1}} \sin \Phi_{1}+p_{33} \sqrt{2 J_{2}} \cos \Phi_{2} \\
y^{\prime}=p_{41} \sqrt{2 J_{1}} \cos \Phi_{1}-p_{42} \sqrt{2 J_{1}} \sin \Phi_{1}+p_{43} \sqrt{2 J_{2}} \sin \Phi_{2}-p_{44} \sqrt{2 J_{2}} \sin \Phi_{2}
\end{array}\right.
$$

Eq. (13) is a general expression to $\left(x, x^{\prime}, y, y^{\prime}\right)$ coordinates of one particle's motion. It has very clear physical meanings, and it is convenient for the experimental BPM turn-by-turn data interpretations. And in the betatron phase rotation frame, the eigentunes, the eigenmodes' initial phases and the eigenmodes' phase advances are clearly defined. The particle oscillation energy and two eigenmodes' action are also well defined. In the following, we will see that Eq. (13) is useful for the analytical calculations.

\subsection{Reduce to uncoupled situation}

In the uncoupled situation, all elements in the two off-diagonal blocks of matrix $\mathbf{P}$ will disappear:

$$
\left(\begin{array}{c}
x \\
x^{\prime} \\
y \\
y^{\prime}
\end{array}\right)=\left(\begin{array}{cccc}
p_{11} & 0 & 0 & 0 \\
p_{21} & p_{22} & 0 & 0 \\
0 & 0 & p_{33} & 0 \\
0 & 0 & p_{43} & p_{44}
\end{array}\right) \cdot\left(\begin{array}{c}
\sqrt{2 J_{1}} \cos \Phi_{1} \\
-\sqrt{2 J_{1}} \sin \Phi_{1} \\
\sqrt{2 J_{2}} \cos \Phi_{2} \\
-\sqrt{2 J_{2}} \sin \Phi_{2}
\end{array}\right)
$$

The four coordinates in the laboratory frame are:

$$
\begin{aligned}
& \left\{\begin{aligned}
x & =p_{11} \sqrt{2 J_{1}} \cos \Phi_{1} \\
x^{\prime} & =p_{21} \sqrt{2 J_{1}} \cos \Phi_{1}-p_{22} \sqrt{2 J_{1}} \sin \Phi_{1}
\end{aligned}\right. \\
& \left\{\begin{aligned}
y & =p_{33} \sqrt{2 J_{2}} \cos \Phi_{2} \\
y^{\prime} & =p_{43} \sqrt{2 J_{2}} \cos \Phi_{2}-p_{44} \sqrt{2 J_{2}} \sin \Phi_{2}
\end{aligned}\right.
\end{aligned}
$$

Comparing to the uncoupled one-dimensional Courant-Snyder parameterization:

$$
\begin{aligned}
& \left\{\begin{aligned}
x & =\sqrt{2 J_{x} \beta_{x}} \cos \left(\int \frac{1}{\beta_{x}} d s+\phi_{x, 0}\right) \\
x^{\prime} & =-\alpha_{x} \sqrt{2 J_{x} / \beta_{x}} \cos \left(\int \frac{1}{\beta_{x}} d s+\phi_{x, 0}\right)-\sqrt{2 J_{x} / \beta_{x}} \sin \left(\int \frac{1}{\beta_{x}} d s+\phi_{x, 0}\right)
\end{aligned}\right. \\
& \left\{\begin{array}{r}
y=\sqrt{2 J_{y} \beta_{y}} \cos \left(\int \frac{1}{\beta_{y}} d s+\phi_{y, 0}\right) \\
y^{\prime}=-\alpha_{y} \sqrt{2 J_{y} / \beta_{y}} \cos \left(\int \frac{1}{\beta_{y}} d s+\phi_{y, 0}\right)-\sqrt{2 J_{y} / \beta_{y}} \sin \left(\int \frac{1}{\beta_{y}} d s+\phi_{y, 0}\right)
\end{array},\right.
\end{aligned}
$$


we find the connections of $p_{i j}$ s to Twiss parameters defined in Courant-Synder's one-dimensional parameterization as:

$$
\left\{\begin{array}{l}
p_{11}=\sqrt{\beta_{x}} \\
p_{21}=-\alpha_{x} \sqrt{\beta_{x}} \\
p_{22}=1 / \sqrt{\beta_{x}} \\
p_{33}=\sqrt{\beta_{y}} \\
p_{43}=-\alpha_{y} \sqrt{\beta_{y}} \\
p_{44}=1 / \sqrt{\beta_{y}}
\end{array} .\right.
$$

And the betatron phase connections are:

$$
\left\{\begin{array}{l}
\Phi_{1}=\int \frac{1}{\beta_{x}} d s+\phi_{x, 0} \\
\Phi_{2}=\int \frac{1}{\beta_{y}} d s+\phi_{y, 0}
\end{array}\right.
$$

which coincides with our previous definitions.

\subsection{One eigenmode excitation}

In order to measure the betatron oscillation optics, we coherently shake the beam with one eigenmode's frequency $[11,20]$. If only mode I is activated, the oscillation of the center of the bunch is:

$$
\left(\begin{array}{c}
x \\
x^{\prime} \\
y \\
y^{\prime}
\end{array}\right)=\left(\begin{array}{cccc}
p_{11} & 0 & p_{13} & p_{14} \\
p_{21} & p_{22} & p_{23} & p_{24} \\
p_{31} & p_{32} & p_{33} & 0 \\
p_{41} & p_{42} & p_{43} & p_{44}
\end{array}\right) \cdot\left(\begin{array}{c}
\sqrt{2 J_{1}} \cos \Phi_{1} \\
-\sqrt{2 J_{1}} \sin \Phi_{1} \\
0 \\
0
\end{array}\right),
$$

each coordinate in the laboratory frame is given by:

$$
\left\{\begin{array}{rl}
x & =p_{11} \sqrt{2 J_{1}} \cos \Phi_{1} \\
x^{\prime} & =p_{21} \sqrt{2 J_{1}} \cos \Phi_{1}-p_{22} \sqrt{2 J_{1}} \sin \Phi_{1} \\
y & =p_{31} \sqrt{2 J_{1}} \cos \Phi_{1}-p_{32} \sqrt{2 J_{1}} \sin \Phi_{1} \\
y^{\prime} & =p_{41} \sqrt{2 J_{1}} \cos \Phi_{1}-p_{42} \sqrt{2 J_{1}} \sin \Phi_{1}
\end{array} .\right.
$$

If only mode II is activated, the oscillation of the center of the bunch is given by:

$$
\left(\begin{array}{c}
x \\
x^{\prime} \\
y \\
y^{\prime}
\end{array}\right)=\left(\begin{array}{cccc}
p_{11} & 0 & p_{13} & p_{14} \\
p_{21} & p_{22} & p_{23} & p_{24} \\
p_{31} & p_{32} & p_{33} & 0 \\
p_{41} & p_{42} & p_{43} & p_{44}
\end{array}\right) \cdot\left(\begin{array}{c}
0 \\
0 \\
\sqrt{2 J_{2}} \cos \Phi_{2} \\
-\sqrt{2 J_{2}} \sin \Phi_{2}
\end{array}\right),
$$

each coordinate in the laboratory frame is given by:

$$
\left\{\begin{aligned}
x & =p_{13} \sqrt{2 J_{2}} \cos \Phi_{2}-p_{14} \sqrt{2 J_{2}} \sin \Phi_{2} \\
x^{\prime} & =p_{23} \sqrt{2 J_{2}} \cos \Phi_{2}-p_{24} \sqrt{2 J_{2}} \sin \Phi_{2} \\
y & =p_{33} \sqrt{2 J_{2}} \cos \Phi_{2} \\
y^{\prime} & =p_{43} \sqrt{2 J_{2}} \cos \Phi_{2}-p_{44} \sqrt{2 J_{2}} \sin \Phi_{2}
\end{aligned}\right.
$$

So mode I's betatron phase is reflected in $x$ coordinate when the beam is shaked with mode I's frequency, the mode II's betatron phase is reflected in $y$ coordinate when the beam is shaked with mode II's frequency. The phase in $y$ displacement with mode I frequency shaking, or the phase in $x$ displacement with mode II frequency shaking reflects the off-diagonal terms of matrix $\mathbf{P}$.

P. Bagley [11], R. Talman [21], G. Bourianoff [22] also derived $x$ and $y$ coordinates under one eigenmode excitation. Twiss parameters and/or normalized coupling matrix $\overline{\mathbf{C}}$ are used. Comparing to their expressions, Eq. (22) and Eq. (24) are simple, which is only one specification to the general expression Eq. (13).

\subsection{Matrix $\mathrm{P}$ and $\mathrm{T}_{1 \rightarrow 2}$}

The coordinates in the laboratory frame at $s_{2}$ can be obtained from those at $s_{1}$ by:

$$
\left(\begin{array}{c}
x \\
x^{\prime} \\
y \\
y^{\prime}
\end{array}\right)_{2}=\mathbf{T}_{\mathbf{1 \rightarrow 2}} \cdot\left(\begin{array}{c}
x \\
x^{\prime} \\
y \\
y^{\prime}
\end{array}\right)_{\mathbf{1}}
$$


or through the betatron phase rotation frame:

$$
\left(\begin{array}{c}
x \\
x^{\prime} \\
y \\
y^{\prime}
\end{array}\right)_{2}=\mathbf{P}_{\mathbf{2}} \cdot \mathbf{R}\left(\boldsymbol{\Delta} \boldsymbol{\Phi}_{\mathbf{1}}, \boldsymbol{\Delta} \boldsymbol{\Phi}_{\mathbf{2}}\right) \cdot \mathbf{P}_{\mathbf{1}}^{-} \cdot\left(\begin{array}{c}
x \\
x^{\prime} \\
y \\
y^{\prime}
\end{array}\right)_{\mathbf{1}} .
$$

So we obtain:

$$
\mathbf{T}_{1 \rightarrow 2}=\mathbf{P}_{2} \cdot \mathbf{R}\left(\Delta \Phi_{1}, \Delta \Phi_{2}\right) \cdot \mathbf{P}_{1}^{-} .
$$

If the phase advances between the two points are known, then we can calculate matrix $\mathbf{P}_{\mathbf{2}}$ with the section transfer map $\mathbf{T}_{\mathbf{1} \rightarrow \mathbf{2}}$ and matrix $\mathbf{P}_{\mathbf{1}}$ :

$$
P_{2}=T_{1 \rightarrow 2} \cdot P_{1} \cdot R^{-}\left(\Delta \Phi_{1}, \Delta \Phi_{2}\right)
$$

For the one-turn transfer map, we obtain:

$$
\mathbf{T}=\mathbf{P} \cdot \mathbf{R}\left(2 \pi \mu_{1}, 2 \pi \mu_{2}\right) \cdot \mathbf{P}^{-} .
$$

\section{Construction of matrix $\mathbf{P}$}

In this section, we discuss how to construct the matrix $\mathbf{P}$ analytically and experimentally. It will be found that the matrix $\mathbf{P}$ can be obtained from the eigenvectors of the one-turn transfer map $\mathbf{T}$ in the laboratory frame, and has tight connections with Twiss and coupling parameters defined in Edwards-Teng's parameterization.

\subsection{Matrix $\mathrm{P}$ and eigenvectors}

We assume the four eigenvectors of the one-turn transfer matrix $\mathbf{T}$ are: $\mathbf{v}_{\mathbf{1}}, \mathbf{v}_{\mathbf{1}}^{*}, \mathbf{v}_{\mathbf{2}}, \mathbf{v}_{\mathbf{2}}^{*}$. Their eigenvalues $\lambda_{i}, i=1,2,3,4$, are $e^{i 2 \pi \mu_{1}}, e^{-i 2 \pi \mu_{1}}, e^{i 2 \pi \mu_{2}}, e^{-i 2 \pi \mu_{2}}$, respectively.

$$
\left\{\begin{array}{l}
\mathbf{T} \cdot \mathbf{v}_{\mathbf{1}}=e^{i 2 \pi \mu_{1}} \cdot \mathbf{v}_{\mathbf{1}} \\
\mathbf{T} \cdot \mathbf{v}_{\mathbf{1}}^{*}=e^{-i 2 \pi \mu_{1}} \cdot \mathbf{v}_{\mathbf{1}}^{*} \\
\mathbf{T} \cdot \mathbf{v}_{\mathbf{2}}=e^{i 2 \pi \mu_{2}} \cdot \mathbf{v}_{\mathbf{2}} \\
\mathbf{T} \cdot \mathbf{v}_{\mathbf{2}}^{*}=e^{-i 2 \pi \mu_{2}} \cdot \mathbf{v}_{\mathbf{2}}^{*}
\end{array}\right.
$$

Substituting Eq. (29) into the above equations, after some matrix manipulations, we get:

$$
\left\{\begin{array}{l}
\mathbf{R}\left(\mathbf{2} \pi \mu_{\mathbf{1}}, \mathbf{2} \pi \mu_{\mathbf{2}}\right) \cdot\left(\mathbf{P}^{-} \cdot \mathbf{v}_{\mathbf{1}}\right)=e^{i 2 \pi \mu_{1}} \cdot\left(\mathbf{P}^{-} \cdot \mathbf{v}_{\mathbf{1}}\right) \\
\mathbf{R}\left(\mathbf{2} \pi \mu_{\mathbf{1}}, \mathbf{2} \pi \mu_{\mathbf{2}}\right) \cdot\left(\mathbf{P}^{-} \cdot \mathbf{v}_{\mathbf{1}}^{*}\right)=e^{-i 2 \pi \mu_{1}} \cdot\left(\mathbf{P}^{-} \cdot \mathbf{v}_{\mathbf{1}}^{*}\right) \\
\mathbf{R}\left(\mathbf{2} \pi \mu_{\mathbf{1}}, \mathbf{2} \pi \mu_{\mathbf{2}}\right) \cdot\left(\mathbf{P}^{-} \cdot \mathbf{v}_{\mathbf{2}}\right)=e^{i 2 \pi \mu_{2}} \cdot\left(\mathbf{P}^{-} \cdot \mathbf{v}_{\mathbf{2}}\right) \\
\mathbf{R}\left(\mathbf{2} \pi \mu_{\mathbf{1}}, \mathbf{2} \pi \mu_{\mathbf{2}}\right) \cdot\left(\mathbf{P}^{-} \cdot \mathbf{v}_{\mathbf{2}}^{*}\right)=e^{-i 2 \pi \mu_{2}} \cdot\left(\mathbf{P}^{-} \cdot \mathbf{v}_{\mathbf{2}}^{*}\right)
\end{array} .\right.
$$

We normalize the eigenvectors of the one-turn transfer map $\mathbf{T}$ according to:

$$
\mathbf{v}_{1,2}^{\mathbf{T}} \cdot \mathbf{v}_{1,2}^{*}=1
$$

However, after the normalization, $\mathbf{v}_{\mathbf{1 , 2}}$ and $\mathbf{v}_{\mathbf{1}, \mathbf{2}} \cdot \mathbf{e}^{\mathbf{i} \phi}$ both meet Eq. (32), and have the same eigenvalues. In order to assure $p_{12}$ and $p_{34}$ in the matrix $\mathbf{P}$ equal to zero, here we will force $\mathbf{v}_{\mathbf{1}}$ and $\mathbf{v}_{\mathbf{1}}^{*}$ 's first elements to be purely imaginary numbers. Their amplitudes will not be changed. The reason for that will be clear in the following when we connect matrix $\mathbf{P}$ to the eigenvectors of the one-turn transfer map $\mathbf{T}$.

If the phase of the first element of $\mathbf{v}_{\mathbf{1}}$ is $e^{i \theta_{1}}$, we multiply $\mathbf{v}_{\mathbf{1}}$ by $e^{i(-\pi / 2-\theta)}$. Then the phase of the first element of $\mathbf{v}_{\mathbf{1}}^{*}$ is $e^{-i \theta_{1}}$, we multiply $\mathbf{v}_{\mathbf{1}}^{*}$ by $e^{i\left(\pi / 2+\theta_{1}\right)}$. The same procedure is needed for the eigenvectors $\mathbf{v}_{\mathbf{2}}$ and $\mathbf{v}_{\mathbf{2}}^{*}$. If the phase of the third element of $\mathbf{v}_{\mathbf{2}}$ is $e^{i \theta_{2}}$, we multiply $\mathbf{v}_{\mathbf{2}}$ by $e^{i\left(-\pi / 2-\theta_{2}\right)}$. Then the phase of the third element of $\mathbf{v}_{\mathbf{2}}^{*}$ is $e^{-i \theta_{2}}$, we multiply $\mathbf{v}_{\mathbf{2}}^{*}$ by $e^{i\left(\pi / 2+\theta_{2}\right)}$. After that the first elements of $\mathbf{v}_{\mathbf{1}}$ and $\mathbf{v}_{\mathbf{1}}^{*}$, the third elements of $\mathbf{v}_{\mathbf{2}}$ and $\mathbf{v}_{\mathbf{2}}^{*}$ are all purely imaginary numbers.

For the rotation matrix $\mathbf{R}\left(\mathbf{2} \pi \mu_{1}, \mathbf{2} \pi \mu_{2}\right)$, there are also four normalized eigenvectors. Their eigenvalues $\lambda_{i}, i=1,2,3,4$, are $e^{i 2 \pi \mu_{1}}, e^{-i 2 \pi \mu_{1}}, e^{i 2 \pi \mu_{2}}, e^{-i 2 \pi \mu_{2}}$, respectively. The normalized eigenvectors of the matrix $\mathbf{R}\left(\mathbf{2} \pi \mu_{1}, \mathbf{2} \pi \mu_{\mathbf{2}}\right)$ are $\tilde{\mathbf{v}}_{1}, \tilde{\mathbf{v}}_{1}^{*}, \tilde{\mathbf{v}}_{2}, \tilde{\mathbf{v}}_{2}^{*}$ :

$$
\left\{\begin{array}{l}
\tilde{\mathbf{v}}_{1}=\frac{1}{\sqrt{2}}\left(\begin{array}{llll}
-\mathrm{i} & 1 & 0 & 0
\end{array}\right)^{T} \\
\tilde{\mathbf{v}}_{1}^{*}=\frac{1}{\sqrt{2}}\left(\begin{array}{lllll}
\mathrm{i} & 1 & 0 & 0
\end{array}\right)^{T} \\
\tilde{\mathbf{v}}_{2}=\frac{1}{\sqrt{2}}\left(\begin{array}{llll}
0 & 0 & -\mathrm{i} & 1
\end{array}\right)^{T} \\
\tilde{\mathbf{v}}_{2}^{*}=\frac{1}{\sqrt{2}}\left(\begin{array}{llll}
0 & 0 & \mathrm{i} & 1
\end{array}\right)^{T}
\end{array}\right.
$$




$$
\left\{\begin{array}{l}
\mathbf{R}\left(\mathbf{2} \pi \mu_{\mathbf{1}}, \mathbf{2} \pi \mu_{\mathbf{2}}\right) \cdot \tilde{\mathbf{v}}_{\mathbf{1}}=e^{i 2 \pi \mu_{1}} \cdot \tilde{\mathbf{v}}_{1} \\
\mathbf{R}\left(\mathbf{2} \pi \mu_{\mathbf{1}}, \mathbf{2} \pi \mu_{\mathbf{2}}\right) \cdot \tilde{\mathbf{v}}_{\mathbf{1}}^{*}=e^{-i 2 \pi \mu_{1}} \cdot \tilde{\mathbf{v}}_{1}^{*} \\
\mathbf{R}\left(\mathbf{2} \pi \mu_{\mathbf{1}}, \mathbf{2} \pi \mu_{\mathbf{2}}\right) \cdot \tilde{\mathbf{v}}_{\mathbf{2}}=e^{i 2 \pi \mu_{2}} \cdot \tilde{\mathbf{v}}_{2} \\
\mathbf{R}\left(\mathbf{2} \pi \mu_{\mathbf{1}}, \mathbf{2} \pi \mu_{\mathbf{2}}\right) \cdot \tilde{\mathbf{v}}_{\mathbf{2}}^{*}=e^{-i 2 \pi \mu_{2}} \cdot \tilde{\mathbf{v}}_{2}^{*}
\end{array}\right.
$$

Comparing Eq. (31) and Eq. (34), we obtain:

$$
\left\{\begin{array}{l}
\mathbf{P}^{-} \cdot \mathbf{v}_{\mathbf{1}}=\frac{1}{\sqrt{2}}\left(\begin{array}{llll}
-\mathrm{i} & 1 & 0 & 0
\end{array}\right)^{T} \\
\mathbf{P}^{-} \cdot \mathbf{v}_{\mathbf{1}}^{*}=\frac{1}{\sqrt{2}}\left(\begin{array}{llll}
\mathrm{i} & 1 & 0 & 0
\end{array}\right)^{T} \\
\mathbf{P}^{-} \cdot \mathbf{v}_{\mathbf{2}}=\frac{1}{\sqrt{2}}\left(\begin{array}{lllll}
0 & 0 & -\mathrm{i} & 1
\end{array}\right)^{T} \\
\mathbf{P}^{-} \cdot \mathbf{v}_{\mathbf{2}}^{*}=\frac{1}{\sqrt{2}}\left(\begin{array}{llll}
0 & 0 & \mathrm{i} & 1
\end{array}\right)^{T}
\end{array}\right.
$$

The eigenvectors of matrix $\mathbf{T}$ and $\mathbf{R}\left(\mathbf{2} \pi \mu_{1}, \mathbf{2} \pi \mu_{\mathbf{2}}\right)$ are all complex. Here we recombine the above four equations:

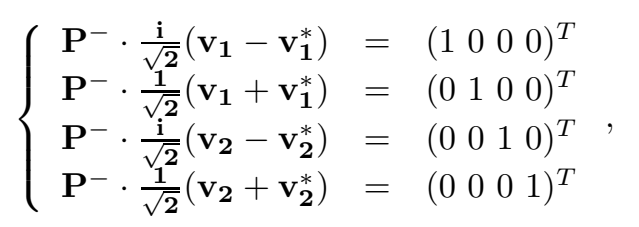

Defining four new vectors from the eigenvectors of matrix $\mathbf{T}$ :

$$
\left\{\begin{array}{l}
\mathbf{e}_{\mathbf{1}}=\frac{i}{\sqrt{2}}\left(\mathbf{v}_{\mathbf{1}}-\mathbf{v}_{\mathbf{1}}^{*}\right) \\
\mathbf{e}_{\mathbf{2}}=\frac{1}{\sqrt{2}}\left(\mathbf{v}_{\mathbf{1}}+\mathbf{v}_{\mathbf{1}}^{*}\right) \\
\mathbf{e}_{\mathbf{3}}=\frac{i}{\sqrt{2}}\left(\mathbf{v}_{\mathbf{2}}-\mathbf{v}_{\mathbf{2}}^{*}\right) \\
\mathbf{e}_{\mathbf{4}}=\frac{1}{\sqrt{2}}\left(\mathbf{v}_{\mathbf{2}}+\mathbf{v}_{\mathbf{2}}^{*}\right)
\end{array}\right.
$$

then Eq. (36) can be re-written as:

$$
\left\{\begin{array}{l}
\mathbf{P}^{-} \cdot \mathbf{e}_{\mathbf{1}}=\left(\begin{array}{llll}
1 & 0 & 0 & 0
\end{array}\right)^{T} \\
\mathbf{P}^{-} \cdot \mathbf{e}_{\mathbf{2}}=\left(\begin{array}{lllll}
0 & 1 & 0 & 0
\end{array}\right)^{T} \\
\mathbf{P}^{-} \cdot \mathbf{e}_{\mathbf{3}}=\left(\begin{array}{llll}
0 & 0 & 1 & 0
\end{array}\right)^{T} \\
\mathbf{P}^{-} \cdot \mathbf{e}_{\mathbf{4}}=\left(\begin{array}{lllll}
0 & 0 & 0 & 1
\end{array}\right)^{T}
\end{array}\right.
$$

or in a compact way:

$$
\mathbf{P}^{-} \cdot \mathbf{e}=\mathbf{I}
$$

where matrix $\mathbf{e}$ is constructed as:

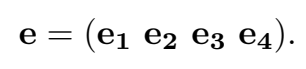

I is $4 \times 4$ identity unit matrix. From Eq. (39), we obtain:

$$
\mathbf{P}=\mathbf{e}
$$

Since P's determinant is 1 , so we should normalize matrix e to make its determinant to be 1 in the end of the procedure.

Then we find that the matrix $\mathbf{P}$ can be directly constructed from the eigenvectors of the one-turn transfer map $\mathbf{T}$ in a sample way. It is easy to check that $p_{12}$ and $p_{34}$ in the matrix $\mathbf{P}$ are both zero from the above procedure.

\subsection{Matrix $\mathrm{P}$ and Twiss, coupling parameters}

Edwards-Teng's parameterization decouples the coupled one-turn transfer map like:

$$
\begin{gathered}
\mathbf{T}=\mathbf{V} \cdot\left(\begin{array}{cc}
\mathbf{A} & 0 \\
0 & \mathbf{B}
\end{array}\right) \cdot \mathbf{V}^{-} \\
\mathbf{V}=\left(\begin{array}{cc}
r \mathbf{I} & \mathbf{C} \\
-\mathbf{C}^{+} & r \mathbf{I}
\end{array}\right)
\end{gathered}
$$


In order to easily distinguish Twiss parameter $\gamma$, the author uses $r$ in the matrix $\mathbf{V}$ instead of $\gamma$ in other literatures. Each matrix A and matrix B are parameterized like one-dimensional Courant-Snyder parameterization [1]. Matrix $\mathbf{A}$ and matrix $\mathbf{B}$ can be further parameterized as $\mathbf{U}_{\mathbf{i}} \cdot \mathbf{R}\left(\mathbf{2} \pi \mu_{\mathbf{i}}\right) \cdot \mathbf{U}_{\mathbf{i}}^{-}$:

$$
\mathbf{U}_{\mathbf{i}}=\left(\begin{array}{cc}
\sqrt{\beta_{i}} & 0 \\
-\alpha_{i} / \sqrt{\beta_{i}} & 1 / \sqrt{\beta_{i}}
\end{array}\right)
$$

Then the one-turn map $\mathbf{T}$ is:

$$
\begin{gathered}
\mathbf{T}=\mathbf{V} \cdot \mathbf{U} \cdot \mathbf{R}\left(\mathbf{2} \pi \mu_{1}, \mathbf{2} \pi \mu_{\mathbf{2}}\right) \cdot \mathbf{U}^{-} \cdot \mathbf{V}^{-}, \\
\mathbf{U}=\left(\begin{array}{cc}
\mathbf{U}_{1} & 0 \\
0 & \mathbf{U}_{2}
\end{array}\right) .
\end{gathered}
$$

Comparing to Eq. (29) and Eq. (45), we obtain:

$$
\begin{gathered}
\mathbf{P}=\mathbf{V} \cdot \mathbf{U}, \\
\|\mathbf{P}\|=\|\mathbf{V}\| \cdot\|\mathbf{U}\|=\mathbf{1} .
\end{gathered}
$$

It is interesting that the matrix $\mathbf{P}$ is the same as the matrix $\mathbf{V} \cdot \mathbf{U}$ defined in Edwards-Teng's parameterization. Expanding $\mathbf{V} \cdot \mathbf{U}$ into Twiss and coupling parameters defined in Edwards-Teng's parameterization, we obtain:

$$
\begin{gathered}
\mathbf{P}=\left(\begin{array}{cc}
\mathbf{P 1 1} & \mathbf{P 1 2} \\
\mathbf{P 2 1} & \mathbf{P 2 2}
\end{array}\right)=\left(\begin{array}{ccc}
r \mathbf{U}_{\mathbf{1}} & \mathbf{C U}_{\mathbf{2}} \\
-\mathbf{C}^{+} \mathbf{U}_{\mathbf{1}} & r \mathbf{U}_{\mathbf{2}}
\end{array}\right) . \\
\mathbf{P}=\left(\begin{array}{cccc}
r \sqrt{\beta_{1}} & 0 & c_{11} \sqrt{\beta_{2}}-c_{12} \alpha_{2} / \sqrt{\beta_{2}} & -c_{12} / \sqrt{\beta_{2}} \\
-\alpha_{1} r / \sqrt{\beta_{1}} & r / \sqrt{\beta_{1}} & c_{21} \sqrt{\beta_{2}}-c_{22} \alpha_{2} / \sqrt{\beta_{2}} & c_{22} / \sqrt{\beta_{2}} \\
-c_{12} \alpha_{1} / \sqrt{\beta_{1}}-c_{22} \sqrt{\beta_{1}} & c_{12} / \sqrt{\beta_{1}} & r \sqrt{\beta_{2}} & 0 \\
c_{11} \alpha_{1} / \sqrt{\beta_{1}}+c_{21} \sqrt{\beta_{1}} & -c_{11} / \sqrt{\beta_{1}} & -\alpha_{2} r / \sqrt{\beta_{2}} & r / \sqrt{\beta_{2}}
\end{array}\right) .
\end{gathered}
$$

The matrix $\mathbf{P}$ is independent of Edwards-Teng's parameterization. However, since Edwards-Teng's parameterization gives the well-defined Twiss parameters and the coupling matrix $\mathbf{C}$, we would like directly borrow them into the betatron phase rotation parameterization.

On the other hand, we can calculate Twiss parameters and coupling parameters from matrix $\mathbf{P}$. For example, the two eigenmodes' Twiss parameters and $r$ can be obtained in the following equations:

$$
\left\{\begin{aligned}
r & =\sqrt{p_{11} \cdot p_{22}}=\sqrt{p_{33} \cdot p_{44}} \\
\beta_{1} & =p_{11} / p_{22} \\
\alpha_{1} & =-p_{21} / p_{22} \\
\gamma_{1} & =\left(1+\alpha_{1}^{2}\right) / \beta_{1}=\left(p_{21}^{2}+p_{22}^{2}\right) / p_{11} p_{22} \\
\beta_{2} & =p_{33} / p_{44} \\
\alpha_{2} & =-p_{43} / p_{44} \\
\gamma_{2} & =\left(1+\alpha_{2}^{2}\right) / \beta_{2}=\left(p_{43}^{2}+p_{44}^{2}\right) / p_{33} p_{44}
\end{aligned}\right.
$$

The coupling matrix $\mathbf{C}$ can be obtained, for example, through $\mathbf{P 1 2}$ and $\mathbf{U}_{\mathbf{2}}$ :

$$
\mathrm{C}=\mathbf{P} 12 \cdot \mathrm{U}_{2}^{-}=\mathbf{P} 12 \cdot \mathbf{P} 22^{-} / \mathbf{r}
$$

For the one-turn map there are ten independents. For the matrix $\mathbf{P}$ there are eight independents since two independents $\mu_{1}$ and $\mu_{2}$ have been split ed out. There are different ways to define those eight independents of the matrix $\mathbf{P}$.

\subsection{Construct matrix $\mathbf{P}$ experimentally}

To fully construct matrix $\mathbf{P}$, we need to know the turn-by-turn $\left(x, x^{\prime}, y, y^{\prime}\right)$ data at one point in the ring. In each interaction region of the Relativistic Heavy Ion Collider ( RHIC ), there are two DXBPMs which are close to the last magnet DX and face to the IP. There is no other magnet between the two BPMs if we ignore or switch off the detector's solenoid field, so we obtain the turn-by-turn angle informations of the particle's motion at the two BPMs.

Here we give an example of the matrix $\mathbf{P}$ construction through the simulation data. The two DXBPMs in the IR8 in the Blue ring are used to get the angle information. The design distance from the two BPMs to the design IP is $8.33 \mathrm{~m}$. We designate the DXBPM in the upstream of IP8 as DXBPM81, and the DXBPM 
in the downstream of IP82 as DXBPM82. In the following we use SAD code [23] to produce the BPM turn-by-turn data. In the simulation, only one free oscillation particle circulates in the Blue ring.

The two eigentunes can be precisely obtained from the turn-by-turn $x$ and/or $y$ data with the fast Fourier transformation( FFT ) technique. The uncoupled tunes are $\mu_{x, 0}=28.22, \mu_{y, 0}=29.23$. We set the skew quadrupole family 1 's integrated strength to be $0.0005 \mathrm{~m}^{-1}$ to introduce the coupling. From FFT of $(x+y)$ turn-by-turn data at DXBPM81, the two tunes are obtained 28.2126, 29.2375, respectively.

\subsubsection{Initial phases}

Knowing the two eigentunes $\mu_{1}$ and $\mu_{2}$, we first obtain the initial phases of two eigenmodes. Multiplying $\cos \left(2 \pi \mu_{1}(n-1)\right), \sin \left(2 \pi \mu_{1}(n-1)\right)$ to $x$ turn-by-turn data, and taking summations to the maximum Nth turn, we get:

$$
\left\{\begin{array}{c}
C_{1}=\sum_{i=1}^{N} x_{i} \cdot \cos \left(2 \pi \mu_{1}(n-1)\right) \\
S_{1}=\sum_{i=1}^{N} x_{i} \cdot \sin \left(2 \pi \mu_{1}(n-1)\right)
\end{array},\right.
$$

$\phi_{1,0}$ is given by:

$$
\left\{\begin{array}{ccc}
\sin \phi_{1,0} & = & -S_{1} / \sqrt{S_{1}^{2}+C_{1}^{2}} \\
\cos \phi_{1,0} & = & C_{1} / \sqrt{S_{1}^{2}+C_{1}^{2}} \\
\phi_{1,0} & = & \arctan \left(-S_{1} / C_{1}\right) .
\end{array} .\right.
$$

The same procedure to $y$ turn-by-turn BPM data, we get:

$$
\left\{\begin{array}{c}
C_{2}=\sum_{i=1}^{N} y_{i} \cdot \cos \left(2 \pi \mu_{2}(n-1)\right) \\
S_{2}=\sum_{i=1}^{N} y_{i} \cdot \sin \left(2 \pi \mu_{2}(n-1)\right)
\end{array},\right.
$$

the $\phi_{2,0}$ is given by:

$$
\left\{\begin{array}{ccc}
\sin \phi_{2,0} & = & -S_{2} / \sqrt{S_{2}^{2}+C_{2}^{2}} \\
\cos \phi_{2,0} & = & C_{2} / \sqrt{S_{2}^{2}+C_{2}^{2}} \\
\phi_{2,0} & = & \arctan \left(-S_{2} / C_{2}\right) .
\end{array} .\right.
$$

From $x$ turn-by-turn data at DXBPM81, we get mode I's betatron oscillation initial phase $138.6449^{\circ}$. From $y$ turn-by-turn data from DXBPM81, we get mode II's betatron oscillation initial phase $-152.1502^{\circ}$. At DXBPM82, we similarly get mode I and mode II's initial phases $-54.9332^{\circ}$ and $14.0882^{\circ}$. So the eigenmodes' phase advances between the two BPMs are $166.4218^{\circ}$ and $166.2384^{\circ}$ for mode I and mode II, respectively. From the analytical calculation with SAD code, they are $166.4212^{\circ}$ and $166.2399^{\circ}$. Here we see that the phase advances are very precisely reproduced from the simulation turn-by-turn BPM data.

\subsubsection{Matrix A}

Eq. (11) can be rewritten as:

$$
\left(\begin{array}{c}
x \\
x^{\prime} \\
y \\
y^{\prime}
\end{array}\right)=\left(\begin{array}{cccc}
\sqrt{2 J_{1}} p_{11} & -\sqrt{2 J_{1}} p_{12} & \sqrt{2 J_{2}} p_{13} & -\sqrt{2 J_{2}} p_{14} \\
\sqrt{2 J_{1}} p_{21} & -\sqrt{2 J_{1}} p_{22} & \sqrt{2 J_{2}} p_{23} & -\sqrt{2 J_{2}} p_{24} \\
\sqrt{2 J_{1}} p_{31} & -\sqrt{2 J_{1}} p_{32} & \sqrt{2 J_{2}} p_{33} & -\sqrt{2 J_{2}} p_{34} \\
\sqrt{2 J_{1}} p_{41} & -\sqrt{2 J_{1}} p_{42} & \sqrt{2 J_{2}} p_{43} & -\sqrt{2 J_{2}} p_{44}
\end{array}\right) \cdot\left(\begin{array}{c}
\cos \Phi_{1} \\
\sin \Phi_{1} \\
\cos \Phi_{2} \\
\sin \Phi_{2}
\end{array}\right) .
$$

In order to facilitate narration, we define an interim matrix $\mathbf{A}$ as :

$$
\mathbf{A}=\left(\begin{array}{ll}
\mathbf{A 1 1} & \mathbf{A 1 2} \\
\mathbf{A 2 1} & \mathbf{A 2 2}
\end{array}\right)=\left(\begin{array}{llll}
\sqrt{2 J_{1}} p_{11} & -\sqrt{2 J_{1}} p_{12} & \sqrt{2 J_{2}} p_{13} & -\sqrt{2 J_{2}} p_{14} \\
\sqrt{2 J_{1}} p_{21} & -\sqrt{2 J_{1}} p_{22} & \sqrt{2 J_{2}} p_{23} & -\sqrt{2 J_{2}} p_{24} \\
\sqrt{2 J_{1}} p_{31} & -\sqrt{2 J_{1}} p_{32} & \sqrt{2 J_{2}} p_{33} & -\sqrt{2 J_{2}} p_{34} \\
\sqrt{2 J_{1}} p_{41} & -\sqrt{2 J_{1}} p_{42} & \sqrt{2 J_{2}} p_{43} & -\sqrt{2 J_{2}} p_{44}
\end{array}\right) .
$$

we still use harmonic analysis technique to get the coefficients $A_{i j}$. For example, from $x$ turn-by-turn data we get:

$$
\left\{\begin{array}{l}
A_{11}=\left(\sum_{i=1}^{N} x_{i} \cdot \cos \left(2 \pi \mu_{1}(n-1)+\phi_{1,0}\right) \cdot 2 / N\right. \\
A_{12}=\left(\sum_{i=1}^{N} x_{i} \cdot \sin \left(2 \pi \mu_{1}(n-1)+\phi_{1,0}\right) \cdot 2 / N\right. \\
A_{13}=\left(\sum_{i=1}^{N} x_{i} \cdot \cos \left(2 \pi \mu_{2}(n-1)+\phi_{2,0}\right) \cdot 2 / N\right. \\
A_{14}=\left(\sum_{i=1}^{N} x_{i} \cdot \sin \left(2 \pi \mu_{2}(n-1)+\phi_{2,0}\right) \cdot 2 / N\right.
\end{array}\right.
$$


Similarly from $x^{\prime}, y$ and $y^{\prime}$ turn-by-turn data, we obtain $A_{2 j}, A_{3 j}$ and $A_{4 j}, j=1,2,3,4$. From the above $\left(x, x^{\prime}, y, y^{\prime}\right)$ simulation data at DXBPM81, we obtain matrix A:

$$
\mathbf{A}=\left(\begin{array}{cccc}
7.505 \times 10^{-4} & -1.852 \times 10^{-16} & -1.451 \times 10^{-4} & 5.040 \times 10^{-5} \\
-8.898 \times 10^{-5} & -1.061 \times 10^{-5} & 1.790 \times 10^{-5} & -3.912 \times 10^{-6} \\
4.579 \times 10^{-4} & 1.675 \times 10^{-4} & 2.390 \times 10^{-4} & 4.485 \times 10^{-17} \\
-5.187 \times 10^{-5} & -2.634 \times 10^{-5} & -2.831 \times 10^{-5} & -3.419 \times 10^{-6}
\end{array}\right)
$$

\subsubsection{Normalize matrix $P$}

Matrix A includes the action informations. From the determinant of the left upper $2 \times 2$ block and the right lower $2 \times 2$ block of matrix $\mathbf{A}$, we get the ratio of the two actions:

$$
k=\sqrt{J_{1} / J_{2}}=\sqrt{\|\mathbf{A} 11\| /\|\mathbf{A 2 2}\|} .
$$

According to the definition of matrix $\mathbf{A}$, we first invert the signs of the elements of matrix $\mathbf{A}$, then divide the first two column elements by $k$. Normalizing the new matrix's determinant to 1 , then we get the matrix $\mathbf{P}$. Please be advised the order of the procedures.

Based on the above obtained $\mathbf{A}$, we obtain:

$$
\mathbf{P}=\left(\begin{array}{cccc}
6.873 & 1.696 \times 10^{-12} & -4.385 & -1.523 \\
-0.8148 & 0.09712 & 0.5412 & 0.1183 \\
4.193 & -1.534 & 7.225 & -1.356 \times 10^{-12} \\
-0.4750 & 0.2412 & -0.8558 & 0.1033
\end{array}\right)
$$

From matrix $\mathbf{P}$, we can continue to calculate Twiss and coupling parameters, the one-turn map. If we know $\mathbf{P}_{\mathbf{1}}$ and $\mathbf{P}_{\mathbf{2}}$ at two points in the ring, the transfer matrix $\mathbf{T}_{\mathbf{1} \rightarrow \mathbf{2}}$ between them can be obtained. The section transfer map is hopefully useful for the field error diagnostics, on-line optics modeling and so on.

\subsubsection{Construct $\mathbf{P}$ from eigenvectors}

Here we want to take a little time to check the method of the matrix $\mathbf{P}$ construction from the eigenvectors from the eigenvectors of one-turn transfer matrix T. According to Eq. (29), the one-turn transfer map at DXBPM81 is obtained:

$$
\mathbf{T}=\left(\begin{array}{cccc}
8.399 & 69.276 & 0.2010 & 1.0966 \\
-0.9873 & -8.025 & -0.01593 & -0.06211 \\
-0.1946 & -2.1840 & 8.3359 & 69.3290 \\
0.03089 & 0.3202 & -0.9869 & -8.0890
\end{array}\right)
$$

Solve out the eigenvectors and normalize them according to the above given procedures in section 3.1, we re-construct the matrix $\mathbf{P}$ from the eigenvectors of one-turn transfer map $\mathbf{T}$ :

$$
\mathbf{P}=\left(\begin{array}{cccc}
7.035 & 4.308 \times 10^{-16} & -4.284 & 1.488 \\
-0.834 & -0.0994 & 0.5287 & -0.1155 \\
4.292 & 1.570 & 7.0579 & 4.322 \times 10^{-16} \\
-0.486 & -0.247 & -0.836 & -0.101
\end{array}\right)
$$

which is close to the original one Eq. (62).

\section{Phase ellipses and $\Sigma$ matrix}

In the uncoupled one-dimensional situation, it is well-known that the particle traces out an ellipse in the $x-x^{\prime}$ or $y-y^{\prime}$ plane. For the linearly coupled situation, there are two modes' contributions in each laboratory coordinate shown in Eq. (13). There are several ellipses in different projection planes. 


\subsection{Projection in the $x-x^{\prime}$ plane from mode I}

$\left(x, x^{\prime}, y, y^{\prime}\right)$ coordinates from mode $\mathrm{I}$ is :

$$
\left(\begin{array}{c}
x \\
x^{\prime} \\
y \\
y^{\prime}
\end{array}\right)_{I}=\mathbf{P} \cdot\left(\begin{array}{c}
\sqrt{2 J_{1}} \cos \Phi_{1} \\
-\sqrt{2 J_{1}} \sin \Phi_{1} \\
0 \\
0
\end{array}\right)=\left(\begin{array}{ll}
\mathbf{P 1 1} & \mathbf{P 1 2} \\
\mathbf{P 2 1} & \mathbf{P 2 2}
\end{array}\right) \cdot\left(\begin{array}{c}
\sqrt{2 J_{1}} \cos \Phi_{1} \\
-\sqrt{2 J_{1}} \sin \Phi_{1} \\
0 \\
0
\end{array}\right)
$$

The projection in the $x-x^{\prime}$ plane is given:

$$
\left(\begin{array}{c}
x \\
x^{\prime}
\end{array}\right)_{I}=\mathbf{P} 11 \cdot\left(\begin{array}{c}
\sqrt{2 J_{1}} \cos \Phi_{1} \\
-\sqrt{2 J_{1}} \sin \Phi_{1}
\end{array}\right) .
$$

Since $\mathbf{P} 11$ is $2 \times 2$ matrix, its determinant is $r^{2}$, so

$$
\mathbf{P}_{11^{+}}=\mathrm{r}^{2} \cdot \mathbf{P}^{-} 1^{-} .
$$

Multiply $\mathbf{P} 11^{+}$to both sides of Eq. (66), we obtain:

$$
\begin{gathered}
\mathbf{P 1 1}^{+} \cdot\left(\begin{array}{c}
x \\
x^{\prime}
\end{array}\right)_{\mathbf{I}}=\mathbf{r}^{\mathbf{2}} \cdot \sqrt{\mathbf{2 \mathbf { J } _ { \mathbf { 1 } }}} \cdot\left(\begin{array}{c}
\cos \Phi_{1} \\
-\sin \Phi_{1}
\end{array}\right), \\
\left(\begin{array}{cc}
p_{22} & 0 \\
-p_{21} & p_{11}
\end{array}\right) \cdot\left(\begin{array}{c}
x \\
x^{\prime}
\end{array}\right)_{I}=r^{2} \cdot \sqrt{2 J_{1}} \cdot\left(\begin{array}{c}
\cos \Phi_{1} \\
-\sin \Phi_{1}
\end{array}\right) .
\end{gathered}
$$

We normalize $\mathbf{P} 11^{+}$to a unit determinant matrix $\overline{\mathbf{P 1 1}^{+}}$:

$$
\overline{\mathbf{P}_{11}^{+}}=\mathbf{P 1 1}^{+} / \mathbf{r}=\left(\begin{array}{cc}
\overline{p_{22}} & 0 \\
-\overline{p_{21}} & \overline{p_{11}}
\end{array}\right)=\left(\begin{array}{cc}
p_{22} / r & 0 \\
-p_{21} / r & p_{11} / r
\end{array}\right) .
$$

Then we re-write Eq. (68) as:

$$
\overline{\mathbf{P 1 1}^{+}} \cdot\left(\begin{array}{c}
x \\
x^{\prime}
\end{array}\right)_{I}=r \cdot \sqrt{2 J_{1}} \cdot\left(\begin{array}{c}
\cos \Phi_{1} \\
-\sin \Phi_{1}
\end{array}\right)
$$

$\overline{\mathbf{P 1 1}^{+}}$is a $2 \times 2$ matrix, its determinant equals to 1 , so after its transferring, the phase area will not change.

$$
\begin{gathered}
\left(\begin{array}{c}
X \\
X^{\prime}
\end{array}\right)_{I}=\overline{\mathbf{P 1 1}^{+}} \cdot\left(\begin{array}{c}
x \\
x^{\prime}
\end{array}\right)_{I}, \\
\left(\begin{array}{c}
X \\
X^{\prime}
\end{array}\right)_{I}=r \cdot \sqrt{2 J_{1}} \cdot\left(\begin{array}{c}
\cos \Phi_{1} \\
-\sin \Phi_{1}
\end{array}\right),
\end{gathered}
$$

where $\left(X, X^{\prime}\right)$ are the new coordinates. The phase area in $X-X^{\prime}$ plane, or in the $x-x^{\prime}$ plane from mode I's contribution is:

$$
\varepsilon_{I, x-x^{\prime}}=r^{2} \cdot 2 \pi J_{1} .
$$

The projection in the $x-x^{\prime}$ plane from mode I is an ellipse. From Eq. (69), we get:

$$
\left(p_{22} x\right)^{2}+\left(-p_{21} x+p_{22} x^{\prime}\right)^{2}=r^{4} \cdot 2 J_{1},
$$

or in Twiss parameters,

$$
\gamma_{1} x^{2}+2 \alpha_{1} x x^{\prime}+\beta_{1}^{2}{x^{\prime 2}}^{2}=2 J_{1} \cdot r^{2},
$$

Under the uncoupled situation, the above ellipse reduced to:

$$
\gamma_{1} x^{2}+2 \alpha_{1} x x^{\prime}+\beta_{1} x^{\prime 2}=2 J_{1},
$$

which is the same as Courant-Snyder's parameterization [1]. 


\subsection{Projection in the $y-y^{\prime}$ plane from mode I}

Similarly, we calculate the projection in the $y-y^{\prime}$ plane from the contribution of mode I:

$$
\begin{gathered}
\left(\begin{array}{c}
y \\
y^{\prime}
\end{array}\right)_{I}=\mathbf{P} \mathbf{2 1} \cdot\left(\begin{array}{c}
\sqrt{2 J_{1}} \cos \Phi_{1} \\
-\sqrt{2 J_{1}} \sin \Phi_{1}
\end{array}\right) . \\
\mathbf{P}^{+} \cdot\left(\begin{array}{c}
y \\
y^{\prime}
\end{array}\right)_{\mathbf{I}}=\left(\mathbf{1}-\mathbf{r}^{\mathbf{2}}\right) \cdot \sqrt{\mathbf{2 \mathbf { J } _ { 1 }}}\left(\begin{array}{c}
\cos \Phi_{1} \\
-\sin \Phi_{1}
\end{array}\right) .
\end{gathered}
$$

Normalize $\mathbf{P 2 1}^{+}$to a unit determinant matrix $\overline{\mathbf{P 2 1}^{+}}$:

$$
\overline{\mathbf{P}^{+}}=\mathbf{P}^{+} / \sqrt{1-\mathbf{r}^{2}},
$$

then we obtain:

$$
\begin{gathered}
\overline{\mathbf{P 2 1}^{+}} \cdot\left(\begin{array}{c}
y \\
y^{\prime}
\end{array}\right)_{I}=\sqrt{1-r^{2}} \cdot \sqrt{2 J_{1}}\left(\begin{array}{c}
\cos \Phi_{1} \\
-\sin \Phi_{1}
\end{array}\right) . \\
\left(\begin{array}{c}
Y \\
Y^{\prime}
\end{array}\right)_{I}=\sqrt{1-r^{2}} \cdot \sqrt{2 J_{1}}\left(\begin{array}{c}
\cos \Phi_{1} \\
-\sin \Phi_{1}
\end{array}\right) .
\end{gathered}
$$

Then the phase area in the $Y-Y^{\prime}$ plane or in the $y-y^{\prime}$ plane contributed from mode $\mathrm{I}$ is:

$$
\varepsilon_{I, y-y^{\prime}}=\left(1-r^{2}\right) \cdot 2 \pi J_{1} .
$$

The phase shape in the $y-y^{\prime}$ plane is an ellipse. From Eq. (79):

$$
\left(p_{42} y-p_{32} y^{\prime}\right)^{2}+\left(-p_{41} y+p_{31} y^{\prime}\right)^{2}=\left(1-r^{2}\right)^{2} \cdot 2 J_{1} .
$$

Its shape and orientation is decided by the elements of $\mathbf{P 2 1}$, or the coupling matrix $\mathbf{C}$ defined in EdwardsTeng's parameterization.

The total phase area from mode I in the $y-y^{\prime}$ and $x-x^{\prime}$ planes is:

$$
\varepsilon_{I}=\varepsilon_{I, x-x^{\prime}}+\varepsilon_{I, y-y^{\prime}}=2 \pi J_{1} .
$$

We define the phase area partition ratio $\kappa_{I}$ between $x-x^{\prime}$ plane and $y-y^{\prime}$ plane projection phase areas from mode I as:

$$
\kappa_{I}=\varepsilon_{I, y-y^{\prime}} / \varepsilon_{I, x-x^{\prime}}=\left(1-r^{2}\right) / r^{2} .
$$

\subsection{Projection in the $x-y$ plane from mode I}

We also can obtain the projection in the $x-y$ plane from mode I:

$$
\left(\begin{array}{l}
x \\
y
\end{array}\right)_{I}=\left(\begin{array}{cc}
p_{11} & 0 \\
p_{31} & p_{32}
\end{array}\right) \cdot\left(\begin{array}{c}
\sqrt{2 J_{1}} \cos \Phi_{1} \\
-\sqrt{2 J_{1}} \sin \Phi_{1}
\end{array}\right) .
$$

It is easy to prove that the projection phase shape in $x-y$ plane from mode $\mathrm{I}$ is still an ellipse.

$$
\left(p_{32} x\right)^{2}+\left(-p_{31} x+p_{11} y\right)^{2}=\left(c_{12} / r\right)^{2} \cdot r^{4} \cdot 2 J_{1} .
$$

Its shape and orientation are decided by the coupling matrix $\mathbf{C}$.

Its phase area is:

$$
\varepsilon_{I, x-y}=\left|p_{11} p_{32}\right| \cdot 2 \pi J_{1}=\left|c_{12} / r\right| \cdot r^{2} \cdot 2 \pi J_{1} .
$$

If $c_{12}=0$, no phase area into $x-y$ plane.

In the one eigenmode excitation, $x$ and $y$ turn-by-turn coordinates at the dual direction BPMs can be measured, then we can measure some coupling parameters in the $x-y$ projection plane. 


\subsection{Projections from mode II}

Similar to mode I, mode II also has projections in the $y-y^{\prime}, x-x^{\prime}, x-y$ planes. These coordinates from mode II are given by:

$$
\begin{gathered}
\left(\begin{array}{c}
x \\
x^{\prime} \\
y \\
y^{\prime}
\end{array}\right)_{I I}=\mathbf{P} \cdot\left(\begin{array}{c}
0 \\
0 \\
\sqrt{2 J_{2}} \cos \Phi_{2} \\
-\sqrt{2 J_{2}} \sin \Phi_{2}
\end{array}\right)=\left(\begin{array}{cc}
\mathbf{P 1 1} & \mathbf{P 1 2} \\
\mathbf{P 2 1} & \mathbf{P 2 2}
\end{array}\right) \cdot\left(\begin{array}{c}
0 \\
0 \\
\sqrt{2 J_{2}} \cos \Phi_{2} \\
-\sqrt{2 J_{2}} \sin \Phi_{2}
\end{array}\right), \\
\left(\begin{array}{c}
y \\
y^{\prime}
\end{array}\right)_{I I}=\mathbf{P} \mathbf{2 2} \cdot\left(\begin{array}{c}
\sqrt{2 J_{2}} \cos \Phi_{2} \\
-\sqrt{2 J_{2}} \sin \Phi_{2}
\end{array}\right) \\
\left(\begin{array}{c}
x \\
x^{\prime}
\end{array}\right)_{I I}=\mathbf{P 1 2} \cdot\left(\begin{array}{c}
\sqrt{2 J_{2}} \cos \Phi_{2} \\
-\sqrt{2 J_{2}} \sin \Phi_{2}
\end{array}\right) \\
\left(\begin{array}{c}
x \\
y
\end{array}\right)_{I I}=\left(\begin{array}{cc}
p_{13} & p_{14} \\
p_{33} & 0
\end{array}\right) \cdot\left(\begin{array}{c}
\sqrt{2 J_{2}} \cos \Phi_{2} \\
-\sqrt{2 J_{2}} \sin \Phi_{2}
\end{array}\right) .
\end{gathered}
$$

These phase ellipses' areas are obtained:

$$
\left\{\begin{array}{rl}
\varepsilon_{I I, y-y^{\prime}} & =r^{2} \cdot 2 \pi J_{2} \\
\varepsilon_{I I, x-x^{\prime}} & =\left(1-r^{2}\right) \cdot 2 \pi J_{2} \\
\varepsilon_{I I, x-y} & =\left|c_{12} / r\right| \cdot r^{2} \cdot 2 \pi J_{2}
\end{array} .\right.
$$

And we also see the phase projection area of mode II is conservative:

$$
\varepsilon_{I I}=\varepsilon_{I I, y-y^{\prime}}+\varepsilon_{I I, x-x^{\prime}}=2 \pi J_{2}
$$

The phase area partition ratio $\kappa_{I I}$ between the $x-x^{\prime}$ and $y-y^{\prime}$ plane projections from mode II is defined as:

$$
\kappa_{I I}=\varepsilon_{I I, x-x^{\prime}} / \varepsilon_{I I, y-y^{\prime}}=\left(1-r^{2}\right) / r^{2},
$$

then $\kappa_{I}=\kappa_{I I}=\kappa$.

\section{5 $\Sigma$ matrix and tilt angle}

It is interesting to investigate the projections of all particles in one bunch in different projection planes, therefore $\Sigma$ matrix is defined [24]:

$$
\Sigma=\left(\begin{array}{cccc}
\sigma_{x}^{2} & \sigma_{x, x^{\prime}} & \sigma_{x, y} & \sigma_{x, y^{\prime}} \\
\sigma_{x^{\prime}, x} & \sigma_{x^{\prime}}^{2} & \sigma_{x^{\prime}, y} & \sigma_{x^{\prime}, y^{\prime}} \\
\sigma_{y, x} & \sigma_{y, x^{\prime}} & \sigma_{y}^{2} & \sigma_{y, y^{\prime}} \\
\sigma_{y^{\prime}, x} & \sigma_{y^{\prime}, x^{\prime}} & \sigma_{y^{\prime}, y} & \sigma_{y^{\prime}}^{2}
\end{array}\right)=\left(\begin{array}{cccc}
<x^{2}> & <x x^{\prime}> & <x y> & <x y^{\prime}> \\
<x^{\prime} x> & <x^{\prime 2}> & <x^{\prime} y> & <x^{\prime} y^{\prime}> \\
<y x> & <y x^{\prime}> & <y^{2}> & <y y^{\prime}> \\
<y^{\prime} x> & <y^{\prime} x^{\prime}> & <y^{\prime} y> & <y^{\prime 2}>
\end{array}\right) .
$$

Among them, the particles' projection into the $x-y$ planes is more interesting. Using Eq. (13), it is easy to obtain:

$$
\left\{\begin{array}{l}
<x^{2}>=p_{11}^{2}<J_{1}>+p_{13}^{2}<J_{2}>+p_{14}^{2}<J_{2}> \\
<y^{2}>=p_{31}^{2}<J_{1}>+p_{32}^{2}<J_{1}>+p_{33}<J_{2}> \\
<x y>=p_{11} p_{31}<J_{1}>+p_{13} p_{33}<J_{2}>
\end{array} .\right.
$$

Normally the projection of the beam in the $x-y$ plane doesn't like an ellipse since each particle's projection is combination of two ellipses. However, here we still define the coupling tilt angle for one bunch as other literatures [25]:

$$
\tan \Psi=\frac{2<x y>}{\left.<x>^{2}-<y\right\rangle^{2}},
$$

which is valid for $\left\langle x>^{2}-<y>^{2} \neq 0\right.$. The tilt angle is experimentally observable through the synchrotron light monitor for the electron beam when the coupling is weak. 


\section{Propagation of matrix $\mathbf{P}$}

Here we investigate the propagation of matrix $\mathbf{P}$. The phase advances, Twiss parameters and coupling matrix at $s_{2}$ are also given in the elements of the section transfer matrix $\mathbf{T}_{\mathbf{1 \rightarrow 2}}$. Propagation of matrix $\mathbf{P}$ is the key to other parameters' propagations.

\subsection{Matrix P's propagation}

In order to easily distinguish the elements of $\mathbf{P}$ at position $s_{1}$ and $s_{2}$, we denote the quantity at $s_{2}$ has a tilde overhead. The transfer matrix $\mathbf{T}_{\mathbf{1} \rightarrow \mathbf{2}}$ in the laboratory frame from $s_{1}$ to $s_{2}$ is written into $2 \times 2$ block matrix:

$$
\mathbf{T}_{\mathbf{1 \rightarrow 2}}=\left(\begin{array}{cc}
\mathbf{T} 11 & \mathbf{T 1 2} \\
\mathbf{T 2 1} & \mathbf{T 2 2}
\end{array}\right)=\left(\begin{array}{cccc}
t_{11} & t_{12} & t_{13} & t_{14} \\
t_{21} & t_{22} & t_{23} & t_{24} \\
t_{31} & t_{32} & t_{33} & t_{34} \\
t_{41} & t_{42} & t_{43} & t_{44}
\end{array}\right)
$$

According to Eq. (28), the propagation of $\mathbf{P}$ from $s_{1}$ to $s_{2}$ can be re-written as:

$$
\begin{aligned}
& \left(\begin{array}{ll}
\widetilde{\mathrm{P} 11} & \widetilde{\mathrm{P} 12} \\
\widetilde{\mathrm{P} 21} & \widetilde{\mathrm{P} 22}
\end{array}\right)=\left(\begin{array}{ll}
\mathrm{T} 11 & \mathrm{~T} 12 \\
\mathrm{~T} 21 & \mathrm{~T} 22
\end{array}\right) \cdot\left(\begin{array}{ll}
\mathrm{P} 11 & \mathrm{P} 12 \\
\mathrm{P} 21 & \mathrm{P} 22
\end{array}\right) \cdot\left(\begin{array}{cc}
\mathrm{R}^{-}\left(\Delta \Phi_{1}\right) & 0 \\
0 & \mathrm{R}^{-}\left(\Delta \Phi_{2}\right)
\end{array}\right), \\
& \widetilde{\mathbf{P 1 1}}=(\mathbf{T} 11 \cdot \mathbf{P} 11+\mathbf{T} 12 \cdot \mathbf{P} 21) \cdot \mathbf{R}^{-}\left(\Delta \Phi_{1}\right), \\
& \widetilde{\mathbf{P 2 1}}=(\mathbf{T} 21 \cdot \mathbf{P} 11+\mathbf{T} 22 \cdot \mathbf{P 2 1}) \cdot \mathbf{R}^{-}\left(\Delta \Phi_{1}\right), \\
& \widetilde{\mathrm{P} 12}=(\mathrm{T} 11 \cdot \mathrm{P} 12+\mathrm{T} 12 \cdot \mathrm{P} 22) \cdot \mathbf{R}^{-}\left(\Delta \Phi_{2}\right), \\
& \widetilde{\mathbf{P 2 2}}=(\mathbf{T} 21 \cdot \mathbf{P} 12+\mathbf{T} 22 \cdot \mathbf{P} 22) \cdot \mathbf{R}^{-}\left(\Delta \Phi_{2}\right) .
\end{aligned}
$$

From the above equations, if we know the phase advance $\Delta \Phi_{1}, \Delta \Phi_{2}$, the matrix $\mathbf{P}_{2}$ at $s_{2}$ can be obtained with the matrix $\mathbf{P}_{\mathbf{1}}$ and the transfer matrix $\mathbf{T}_{\mathbf{1} \rightarrow \mathbf{2}}$.

\subsection{The phase advances}

As we know the phase advances are independent from particle to particle. So here we investigate one particle which only has mode I 's coordinates at $s_{1}$ in the betatron phase rotation frame, just like one eigenmode excitation. The coordinates in the laboratory frame at $s_{1}$ is:

$$
\left(\begin{array}{c}
x \\
x^{\prime} \\
y \\
y^{\prime}
\end{array}\right)_{1}=\sqrt{2 J_{1}}\left(\begin{array}{c}
p_{11} \cos \Phi_{1} \\
p_{21} \cos \Phi_{1}-p_{22} \sin \Phi_{1} \\
p_{31} \cos \Phi_{1}-p_{32} \sin \Phi_{1} \\
p_{41} \cos \Phi_{1}-p_{42} \sin \Phi_{1}
\end{array}\right) .
$$

The coordinates in the laboratory frame at $s_{2}$ is:

$$
\left(\begin{array}{c}
x \\
x^{\prime} \\
y \\
y^{\prime}
\end{array}\right)_{2}=\sqrt{2 J_{1}}\left(\begin{array}{c}
\widetilde{p_{11}} \cos \left(\Phi_{1}+\Delta \Phi_{1}\right) \\
\widetilde{p_{21}} \cos \left(\Phi_{1}+\Delta \Phi_{1}\right)-\widetilde{p_{22}}\left(\Phi_{1}+\Delta \Phi_{1}\right) \\
\widetilde{p_{31}} \cos \left(\Phi_{1}+\Delta \Phi_{1}\right)-\widetilde{p_{32}} \sin \left(\Phi_{1}+\Delta \Phi_{1}\right) \\
\widetilde{p_{41}} \cos \left(\Phi_{1}+\Delta \Phi_{1}\right)-\widetilde{p_{42}} \sin \left(\Phi_{1}+\Delta \Phi_{1}\right)
\end{array}\right)
$$

From Eq. (25), we obtain:

$$
\left(\begin{array}{c}
\widetilde{p_{11}} \cos \left(\Phi_{1}+\Delta \Phi_{1}\right) \\
\widetilde{p_{21}} \cos \left(\Phi_{1}+\Delta \Phi_{1}\right)-\widetilde{p_{22}} \sin \left(\Phi_{1}+\Delta \Phi_{1}\right) \\
\widetilde{p_{31}} \cos \left(\Phi_{1}+\Delta \Phi_{1}\right)-\widetilde{p_{32}} \sin \left(\Phi_{1}+\Delta \Phi_{1}\right) \\
\widetilde{p_{41}} \cos \left(\Phi_{1}+\Delta \Phi_{1}\right)-\widetilde{p_{42}} \sin \left(\Phi_{1}+\Delta \Phi_{1}\right)
\end{array}\right)=\left(\begin{array}{cccc}
t_{11} & t_{12} & t_{13} & t_{14} \\
t_{21} & t_{22} & t_{23} & t_{24} \\
t_{31} & t_{32} & t_{33} & t_{34} \\
t_{41} & t_{42} & t_{43} & t_{44}
\end{array}\right) \cdot\left(\begin{array}{c}
p_{11} \cos \Phi_{1} \\
p_{21} \cos \Phi_{1}-p_{22} \sin \Phi_{1} \\
p_{31} \cos \Phi_{1}-p_{32} \sin \Phi_{1} \\
p_{41} \cos \Phi_{1}-p_{42} \sin \Phi_{1}
\end{array}\right)
$$

For $\widetilde{p_{11}}$, we obtain:

$$
\begin{aligned}
\widetilde{p_{11}} \cos \left(\Phi_{1}+\Delta \Phi_{1}\right)= & \left(t_{11} p_{11}+t_{12} p_{21}+t_{13} p_{31}+t_{14} p_{41}\right) \cos \Phi_{1} \\
& -\left(t_{12} p_{22}+t_{13} p_{32}+t_{14} p_{42}\right) \sin \Phi_{1}
\end{aligned}
$$


For simplicity, we define:

$$
\begin{gathered}
\mathbf{G}=\mathbf{T}_{\mathbf{1} \rightarrow \mathbf{2}} \cdot \mathbf{P}_{\mathbf{1}}, \\
G_{i j}=\Sigma t_{i k} p_{k j}, k=1,2,3,4 .
\end{gathered}
$$

So $\Delta \Phi_{1}$ is given:

$$
\left\{\begin{array}{c}
\cos \Phi_{1}=G_{11} / \sqrt{G_{11}^{2}+G_{12}^{2}} \\
\sin \Phi_{1}=G_{12} / \sqrt{G_{11}^{2}+G_{12}^{2}} \\
\Delta \Phi_{1}=\arctan \left(G_{12} / G_{11}\right)
\end{array} .\right.
$$

And we also obtain:

$$
\widetilde{p_{11}}=\sqrt{G_{11}^{2}+G_{12}^{2}}
$$

The phase advance $\Delta \Phi_{2}$ for mode II also can be achieved in the above procedure except we assume the particle only have mode II coordinates in the betatron phase rotation frame at $s_{1} . \Delta \Phi_{2}$ and $\widetilde{p_{33}}$ are obtained:

$$
\left\{\begin{aligned}
\cos \Delta \Phi & =G_{33} / \sqrt{G_{33}^{2}+G_{34}^{2}} \\
\sin \Delta \Phi & =G_{34} / \sqrt{G_{33}^{2}+G_{34}^{2}} \\
\Delta \Phi_{2} & =\arctan \left(G_{34} / G_{33}\right) \\
\widetilde{p_{33}} & =\sqrt{G_{33}^{2}+G_{34}^{2}} .
\end{aligned}\right.
$$

\section{$5.3 \widetilde{p_{i j}}$ at $s_{2}$}

Knowing the eigenmodes' betatron oscillation phase advances $\Delta \Phi_{1}$ and $\Delta \Phi_{2}$, according to Eq. (102) to Eq. (105), the matrix $\mathbf{P}_{\mathbf{2}}$ is given:

$$
\begin{aligned}
& \widetilde{\mathbf{P} 11}=\frac{1}{\widetilde{p_{11}}}\left(\begin{array}{ll}
G_{11} & G_{12} \\
G_{21} & G_{22}
\end{array}\right) \cdot\left(\begin{array}{cc}
G_{11} & -G_{12} \\
G_{12} & G_{11}
\end{array}\right), \\
& \widetilde{\mathbf{P 2 1}}=\frac{1}{\widetilde{p_{11}}}\left(\begin{array}{ll}
G_{31} & G_{32} \\
G_{41} & G_{42}
\end{array}\right) \cdot\left(\begin{array}{cc}
G_{11} & -G_{12} \\
G_{12} & G_{11}
\end{array}\right), \\
& \widetilde{\mathbf{P 1 2}}=\frac{1}{\widetilde{p_{33}}}\left(\begin{array}{ll}
G_{13} & G_{14} \\
G_{23} & G_{24}
\end{array}\right) \cdot\left(\begin{array}{cc}
G_{33} & -G_{34} \\
G_{34} & G_{33}
\end{array}\right), \\
& \widetilde{\mathbf{P 2 2}}=\frac{1}{\widetilde{p_{33}}}\left(\begin{array}{ll}
G_{33} & G_{34} \\
G_{43} & G_{44}
\end{array}\right) \cdot\left(\begin{array}{cc}
G_{33} & -G_{34} \\
G_{34} & G_{33}
\end{array}\right) .
\end{aligned}
$$

It is easy to check that the elements $\widetilde{p_{12}}=0, \widetilde{p_{34}}=0$.

\subsection{Twiss and coupling parameters at $s_{2}$}

Then we are able to describe Twiss parameters at $s_{2}$ in these at $s_{1}$ and the transfer matrix $\mathbf{T}_{\mathbf{1} \rightarrow \mathbf{2}}$ 's elements.

$$
\begin{aligned}
& \left\{\begin{array}{l}
\widetilde{\beta_{1}}=\widetilde{p_{11}} / \widetilde{p_{22}}=\frac{G_{11}^{2}+G_{12}^{2}}{G_{11} G_{22}-G_{12} G_{21}} \\
\widetilde{\alpha_{1}}=-\widetilde{p_{21}} / \widetilde{p_{22}}=\frac{G_{11} G_{21}+G_{12} G_{22}}{-G_{11} G_{22}+G_{12} G_{21}}
\end{array},\right. \\
& \left\{\begin{array}{l}
\widetilde{\beta_{2}}=\widetilde{p_{33}} / \widetilde{p_{34}}=\frac{S_{33}^{2}+S_{34}^{2}}{G_{33} G_{44}-G_{34} G_{43}} \\
\widetilde{\alpha_{2}}=-\widetilde{p_{43}} / \widetilde{p_{44}}=\frac{G_{33} G_{43}+G_{34} G_{44}}{-G_{33} G_{44}+G_{34} G_{43}}
\end{array} .\right.
\end{aligned}
$$

The coupling parameter $\tilde{r}$ at $s_{2}$ can be obtained:

$$
\tilde{r}=\widetilde{p_{11}} \cdot \widetilde{p_{22}}=G_{11} G_{22}-G_{12} G_{21},
$$

or

$$
\tilde{r}=\widetilde{p_{33}} \cdot \widetilde{p_{44}}=G_{33} G_{44}-G_{34} G_{43} .
$$

From Eq. (52), Eq. (103), Eq. (104), we get:

$$
\begin{aligned}
\tilde{r} \cdot \tilde{\mathbf{C}} & =\widetilde{\mathbf{P} 12} \cdot \widetilde{\mathbf{P} 22} \\
& =(\mathbf{T} 11 \cdot \mathbf{P} 12+\mathbf{T} 12 \cdot \mathbf{P} 22) \cdot\left(\mathbf{P}^{-} \cdot \mathbf{T}^{-} 1^{-}+\mathbf{T}^{-} \cdot \mathbf{P 2 2}^{-}\right) \\
& =\mathbf{T} 11 \cdot \mathbf{T}^{-} 1^{-}+\mathbf{T} 12 \cdot \mathbf{T} 22^{-}+\mathbf{T} 11 \cdot(\mathbf{r} \cdot \mathbf{C}) \cdot \mathbf{T}^{-} 2^{-}+\mathbf{T} 12 \cdot\left(\mathbf{r} \cdot \mathbf{C}^{-}\right) \cdot \mathbf{T}^{-}
\end{aligned}
$$




\subsection{Reduce to uncoupled situation}

Under the uncoupled situation,

$$
\mathbf{G}=\left(\begin{array}{cccc}
p_{11} t_{11}+p_{21} t_{12} & p_{22} t_{12} & 0 & 0 \\
p_{11} t_{21}+p_{21} t_{22} & p_{22} t_{22} & 0 & 0 \\
0 & 0 & p_{33} t_{33}+p_{43} t_{34} & p_{44} p_{34} \\
0 & 0 & p_{33} t_{43}+p_{43} t_{44} & p_{44} p_{44}
\end{array}\right) .
$$

The phase advances then equal to:

$$
\left\{\begin{array}{l}
\Delta \Phi_{1}=\arctan \left(p_{22} t_{12} /\left(p_{11} t_{11}+p_{21} t_{12}\right)\right) \\
\Delta \Phi_{2}=\arctan \left(p_{44} t_{34} /\left(p_{33} t_{33}+p_{43} t_{34}\right)\right)
\end{array},\right.
$$

or in Twiss parameters at $s_{1}$

$$
\left\{\begin{array}{l}
\Delta \Phi_{1}=\arctan \left(t_{12} /\left(\beta_{1} t_{11}-\alpha_{1} t_{12}\right)\right) \\
\Delta \Phi_{2}=\arctan \left(t_{34} /\left(\beta_{2} t_{33}-\alpha_{2} t_{34}\right)\right)
\end{array} .\right.
$$

$\widetilde{\beta_{1}}$ at $s 2$ is:

$$
\begin{aligned}
\widetilde{\beta_{1}} & =\left(\left(p_{22} t_{12}\right)^{2}+\left(p_{11} t_{11}+p_{21} t_{12}\right)^{2}\right) /\left(p 11 p 22\left(t_{11} t_{22}-t_{12} t_{21}\right)\right) \\
& =\beta_{1} t_{11}^{2}-2 \alpha_{1} t_{11} t_{12}+\gamma_{1} t_{12}^{2} .
\end{aligned}
$$

$\widetilde{\alpha_{1}}$ at $s_{2}$ is:

$$
\begin{aligned}
\widetilde{\alpha_{1}} & =\left(p_{22}^{2} t_{12} t_{22}+\left(p_{11} t_{11}+p_{21} t_{12}\right)\left(p_{11} t_{21}+p_{21} t_{22}\right)\right) /\left(p_{11} p_{22}\left(t_{12} t_{21}-t_{11} t_{22}\right)\right) \\
& =-t_{11} t_{12} \beta_{1}+\left(t_{11} t_{22}+t_{12} t_{21}\right) \alpha_{1}-t_{12} t_{22} \gamma_{1} .
\end{aligned}
$$

All of them are the same to that from the uncoupled one-dimensional situation. For the mode II, we also have similar conclusion.

\subsection{Reduces to uncoupled section}

Under the uncoupled situation, $\mathbf{T} \mathbf{1 2}=\mathbf{0}, \mathbf{T} \mathbf{2 1}=\mathbf{0}$. From Eq. (102), we obtain:

$$
\|\widetilde{\mathbf{P 1 1}}\|=\|\mathbf{P} 11\| \text {. }
$$

Since $\tilde{r}=\|\widetilde{\mathbf{P 1 1}}\|$ and $r=\|\mathbf{P 1 1}\|$, so we obtain that $r$ keeps constant in the uncoupled section. And the propagation of coupling matrix propagation in Eq. (124) reduces to:

$$
\tilde{\mathbf{C}}=\mathbf{T} 11 \cdot \mathbf{C} \cdot \mathbf{T}^{2} 2^{-} .
$$

\subsection{In the interaction region}

In the drifts of interaction regions, from the $\beta$ waist where $\alpha_{i}=0$, we have:

$$
\mathbf{G}=\left(\begin{array}{cccc}
p_{11} & s \cdot p_{22} & p_{13}+s \cdot p_{23} & p_{14}+s \cdot p_{24} \\
0 & p_{22} & p_{23} & p_{24} \\
p_{31}+s \cdot p_{41} & p_{32}+s \cdot p_{42} & p_{33} & s \cdot p_{44} \\
p_{41} & p_{42} & 0 & p_{44}
\end{array}\right) \text {, }
$$

$s$ is the distance from the $\beta$ waist. Then the phase advances from the waist is given:

$$
\left\{\begin{array}{l}
\Delta \Phi_{1}(s)=\arctan \left(s \cdot p_{22} / p_{11}\right)=\arctan \left(s / \beta_{1}\right) \\
\Delta \Phi_{2}(s)=\arctan \left(s \cdot p_{44} / p_{33}\right)=\arctan \left(s / \beta_{2}\right)
\end{array} .\right.
$$

Twiss parameters at $s$ are given:

$$
\left\{\begin{array}{l}
\widetilde{\beta_{1}}=\frac{p_{11}^{2}+\left(s \cdot p_{22}\right)^{2}}{p_{11} p_{22}}=\beta_{1}^{*}+s^{2} / \beta_{1}^{*} \\
\widetilde{\beta_{2}}=\frac{p_{33}^{2}+\left(s \cdot p_{44}\right)^{2}}{p_{33} p_{44}}=\beta_{2}^{*}+s^{2} / \beta_{2}^{*}
\end{array}\right.
$$




$$
\left\{\begin{array}{l}
\widetilde{\alpha_{1}}=-s \cdot \beta_{1}^{*} \\
\widetilde{\alpha_{2}}=-s \cdot \beta_{2}^{*}
\end{array} .\right.
$$

These expressions are same to the uncoupled one-dimensional situation, no matter of the coupling parameters at the $\beta$ waist. The phase advances between the two BPMs close to the IP are used to measure $\beta^{*}$ s at the IP.

\section{Acknowledgments}

The author thanks S. Peggs, R. Talman (Cornell) for the simulating discussions on the local and global coupling observables. Thank D. Trbojevic and T. Roser introduced the author to the topic of the RHIC global decoupling with skew quadrupole modulation technique. And thank S. Peggs and T. Roser for their careful readings and corrections of the draft. During the writing of the article, the author got encouragements from many people and the author would like to thank them. They are Y. Cai(SLAC), A. Chao(SLAC), W. Fischer, D. Trbojevic, F. Zimmerman(CERN). This work is supported by U.S. DOE under contract No. DE-AC02-98CH10886.

\section{References}

[1] E.D. Courant, H.S. Snyder, Ann. Phys. 3 (1958).

[2] G. Ripken, DESY Internal Report: R1-70/04 (1970).

[3] F. Willeke, G. Ripken, CERN Yellow Report 87-03.

[4] H. Wiedemann, Particle Accelerator Physics II, Nonlinear and Higher-Order Beam Dynamics, SpringerVerlag, 1995.

[5] L.C. Teng, NAL Report FN-229, 1971.

[6] D. Edwards, L. Teng, PAC 1973, p.885.

[7] S. Peggs, Particle Accelerator 12, p.219, 1982.

[8] M. Billing, Cornell Report CBN 85-2 ( 1985 ).

[9] D. Sagan, D. Rubin, Phys. Rev. ST Accel. Beam 2, 074001 (1999).

[10] R. Talman, in Lecture Notes in Physics, 343, M. Month and P. Bryant, Editors, 1988

[11] P. Bagley, D. Rubin, PAC 1987, p.1301.

[12] F. C. Iselin, The MAD Program Version 8.13-Physicist Manual, p52, unpublished.

[13] P.castro, et al., PAC 1993.

[14] J. Irwin, C.X. Wang, Y.T. Yan, Phys. Rev. Lett. 82, p.1684 (1999).

[15] C.-x. Wang, V. Sajaev, and C.-Y. Yao, Phys. Rev. ST Accel. Beams 6, 104001(2003)

[16] Y.T. Yan, Y. Cai, et al., SLAC-Pub-10369, 2004.

[17] D. Sagan, Phys. Rev. ST Accel. Beam 4, 122801 (2001).

[18] D. Sagan, R. Meller, et al., Phys. Rev. ST Accel. Beam 3, 102801 (2000).

[19] Y. Cai, Phys. Lett. A. 150, 262 (2003)

[20] J. Borer, C. Bovet, et al., "Harmonic Analysis of Cohenerent Bunch Oscillation in LEP", Proceedings of The Third EPAC, Berlin, Germanny, p1082, 1992.

[21] Talman, "Shaker Response in a Coupled Lattice", 1996, unpublished. 
[22] G. Borianoff, S. Hunt, D. Mathieson, F. Pilat, R. Talman, "Determination of Coupled-Lattice Properties Using Turn-By-Turn Data".

[23] SAD code homepage: http://acc-physics.kek.jp/SAD/sad.html.

[24] K. Brown, in CERN Yellow Report 80-04, 1980.

[25] F. C. Iselin, The MAD Program Version 8.13-Physicist Manual, p49, unpublished. 